\title{
A Convenient and Templated Method for the Fabrication of Monodisperse Micrometer Hollow Titania Spheres
}

\author{
Haibo Yao and Guojun Han \\ Department of Chemistry and Chemical Engineering, Binzhou University, 391, 5th Huanghe Street, Shandong, Binzhou 256603, China \\ Correspondence should be addressed to Haibo Yao; yyhs1981@163.com
}

Received 15 March 2013; Revised 7 June 2013; Accepted 20 June 2013

Academic Editor: Jun Li

Copyright (C) 2013 H. Yao and G. Han. This is an open access article distributed under the Creative Commons Attribution License, which permits unrestricted use, distribution, and reproduction in any medium, provided the original work is properly cited.

\begin{abstract}
A simple and widely applicable methodology was presented to synthesize monodisperse micrometer hollow titania spheres (HTS) based on the templating method. It was performed by using the preformed poly(styrene-acrylic acid) (PSA) as template spheres which was mixed with tetrabutyltitanate (TBOT) in an ethanol solvent under steam treatment. The HTS which were obtained by the calcination of $\mathrm{PSA} / \mathrm{TiO}_{2}$ composite core-shell spheres had a narrow particle size distribution and commendable surface topography characterized by SEM. The calcined HTS at $500^{\circ} \mathrm{C}$ displayed crystalline reflection peaks that were characteristic to the anatase phase by XRD. Moreover, some key influencing factors including TBOT concentration and reaction time were analyzed. As expected, the diameter of HTS could be readily controlled by altering the size of PSA template spheres. In addition, the approach was also applied to fabricate hollow zirconia spheres and other inorganic spheres.
\end{abstract}

\section{Introduction}

Titania has been extensively studied due to the advantages including unique optical, electrical, high refractive index, and chemical attributes. Hollow titania sphere was well known for high porosity, photovoltaic, and photocatalytic properties, along with its applications as catalyst [1], filler [2], whitener [3], UV absorber, photonic crystal [4, 5], and a large bimolecular release system [6]. However, the sensitivity of titania precursors to moisture led to the great difficulties of controlling the precipitation, the particle morphology, and monodispersity $[7,8]$, defining the further wide applications of titania spheres.

A variety of chemical and physicochemical methods have been employed to synthesize HTS, such as nozzle reactor approaches (spray drying or pyrolysis) [9], emulsion/phase separation techniques [10], emulsion/interfacial polymerization strategies [11], self-assembly processes [12], and surface living polymerization [13]. Among these methods, the morphology and microstructures were significantly influenced by the preparative conditions, and the precise optimization of the reaction conditions (concentration of reagents, $\mathrm{pH}$, temperature, and mixing methods) was required to obtain uniform coatings without the occurrence of aggregation of the coated titania particles $[14,15]$. Though many efforts have been made to synthesize HTS, developing an effective way to better control the size and morphology was still a great challenge. Furthermore, until now, the reports about fabricating HTS in micrometer scale were scarce.

Recently, the templating method has been proved as a successful method to fabricate such hollow spherical structure of inorganic materials, such as zinc sulfide-coated polystyrene $[16]$ or silica spheres $[17,18]$ and core-shell latex spheres with semiconductors [19-22]. For the templating method, the apparent advantage was that the shape and cavity size of formed hollow structures were directly determined by template spheres. In the templating method, monodisperse PSA latex was commonly used as colloidal template, because it was readily available in a wide range of sizes. In the previous study [23, 24], a series of micrometer scale monodisperse PSA spheres in dispersion polymerization has been successfully fabricated, which confirmed the high uniformity of titania coating on the PSA template spheres.

In this study, we reported a new, simple, and efficient water vapor-induced hydrolysis route for postsynthesis coating of titania on the preformed positively charged PSA template spheres as shown in Scheme 1. This method was to 


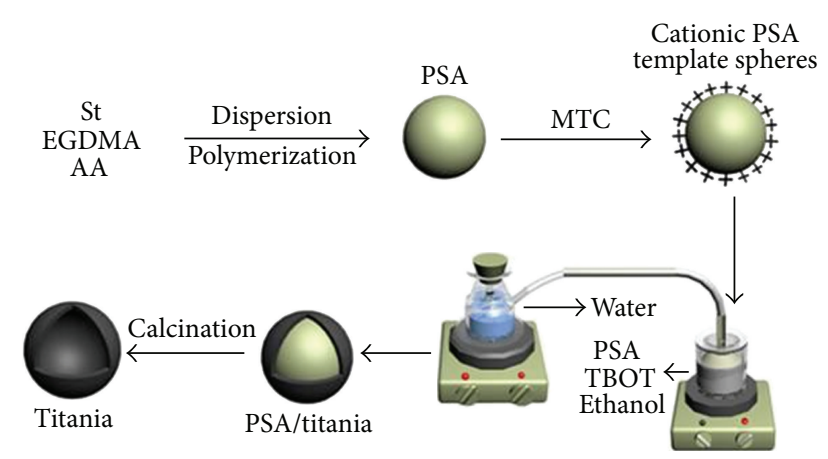

SCHEme 1: Diagram of the formation of HTS.

implement the conventional sol-gel condensation in ethanol solvent. In this procedure, the generated water vapor was bubbled through the TBOT/ethanol solution and impelled the hydrolysis of TBOT to product titania molecules. The generated negative titania molecular precursors deposited on the surface of cationic PSA template spheres to form $\mathrm{PSA} / \mathrm{TiO}_{2}$ composite core-shell spheres. In this case, we did not require careful optimization of the solution conditions to produce uniform coatings on PSA template spheres. Both electrostatic attraction and surface precipitation ensured that the titania molecular precursors were captured by the PSA template spheres, resulting in more uniform nanoparticle coverage. The HTS prepared by this method showed high monodispersity and preferable surface topography. The diameter and the shell thickness of HTS were flexible and tuned by altering the size of PSA template spheres and TBOT concentration, respectively. Furthermore, the zirconia coating hollow spheres with zirconium dichloride oxide $\left(\mathrm{ZrOCl}_{2} \cdot 8 \mathrm{H}_{2} \mathrm{O}\right)$ were also successfully obtained. This approach not only proved to be a facile, low-cost, and environmentally benign way to fabricate HTS but also offered a versatile and effective route for the preparation of other hollow spheres, such as $\mathrm{SiO}_{2}$ and $\mathrm{ZrO}_{2}$.

\section{Experimental}

2.1. Materials. Styrene (St), polyvinylpyrrolidone (PVP), anhydrous ethanol, $\alpha$-methylacrylic acid (AA), 2,2' -azobisisobutyronitrile (AIBN), TBOT, and $\mathrm{ZrOCl}_{2} \cdot 8 \mathrm{H}_{2} \mathrm{O}$ were all purchased from Sinopharm Chemical Reagent Co., Ltd. 2-(Methacryloyl) ethyltrimethylammonium chloride (MTC) and sodium methyl allyl sulfonate (SMAS) were purchased from Zibo Wanduofu Chemical Reagent Co., Ltd. and used as the cationic monomer. Ethylene glycol dimethacrylate (EGDMA) was prepared in our laboratory and used as the crosslinking agent. It was stored at $4^{\circ} \mathrm{C}$ before use. Deionized water was prepared in our laboratory and applied in all polymerization and treatment processes.

2.2. Preparation of Cationic PSA Template Spheres. St, PVP, AA, AIBN, EGDMA, anhydrous ethanol, and water were charged into a $250 \mathrm{~mL}$ three-neck round-bottomed flask which was equipped with a mechanical stirrer, a thermometer, a $\mathrm{N}_{2}$ inlet, a Graham condenser, and a heating mantle. The reaction system was deoxygenated by bubbling nitrogen gas and then heated to $72^{\circ} \mathrm{C}$ under stirred condition. After $5 \mathrm{~h}, \mathrm{MTC}$ was dispersed in anhydrous ethanol and then added into the reaction system. The reaction lasted for $6 \mathrm{~h}$ at $72^{\circ} \mathrm{C}$. The latex emulsion was separated centrifugally and washed twice with ethanol. The formula of fabricating a series of micrometer scale PSA template spheres was presented in the previous study [23]. The obtained white precipitate was air dried to use as follows.

2.3. Preparation of HTS. The fabrication of HTS was shown in Scheme 1. In the typical procedure, water $(30 \mathrm{~mL})$ was placed inside an erlenmeyer of $50 \mathrm{~mL}$ capacity. The prepared cationic PSA powder and TBOT were redispersed into anhydrous ethanol in $50 \mathrm{~mL}$ beaker under gentle stirring. In the subsequent coating process, the water was stirred and heated to $80^{\circ} \mathrm{C}$ for a specified time, and then water vapor that evaporated from the water bath slowly got through the funnel and entered the mixed suspension to react with TBOT, continuously generating white precipitate to deposit on the prepared PSA spheres slowly. After the treatment, the final latex was stirred at ambient temperature until the solvent was fully evaporated. The solid material was washed three times with anhydrous ethanol and heated in a vacuum oven at $90^{\circ} \mathrm{C}$ for $12 \mathrm{~h}$. Finally, the dried material was calcined in a muffle furnace at $500^{\circ} \mathrm{C}$ to generate hollow structure.

In order to investigate the effect of TBOT concentration and reaction time on the forming of HTS, we also performed some contrast experiments following the same procedure mentioned previously. We systematically studied the hollow titania spheres under the condition of different amounts of TBOT and different reaction time $(3 \mathrm{~h}, 9 \mathrm{~h}$, and $15 \mathrm{~h})$. The formula used in this study was as follows: PSA: $1.5 \mathrm{~g}$, absolute ethanol: $20 \mathrm{~mL}$, TBOT: varied from 0.5 to $2.0 \mathrm{~g}$. Furthermore, a series of monodisperse micrometer HTS were fabricated based on altering the size of PSA template spheres referencing to the previous study [23].

2.4. Preparation of Hollow Zirconia Spheres. In the same experimental facility, the different sizes of hollow zirconia spheres with controllable diameters were synthesized following the same procedure mentioned above. In the subsequent process, SMAS was used as anionic surfactant instead of MTC to obtain the anionic PSA spheres, and $\mathrm{ZrOCl}_{2} \cdot 8 \mathrm{H}_{2} \mathrm{O}$ was used as zirconia precursor to generate zirconia in sol-gel process by this approach. Finally, the PSA/ $\mathrm{ZrO}_{2}$ composite spheres were calcined in a muffle furnace to generate hollow zirconia spheres.

2.5. Characterization. The morphology of the products was characterized by SEM (JSM-6380LV). Before the examination, the products were sputtered with gold. TGA was performed using STA 449C instruments. The composite spheres were heated from the ambient temperature to $800^{\circ} \mathrm{C}$ at a rate of $5^{\circ} \mathrm{C} / \mathrm{min}$ under oxygen purge. The infrared spectra were recorded with FTIR (Nicolet IR100) in the range of 


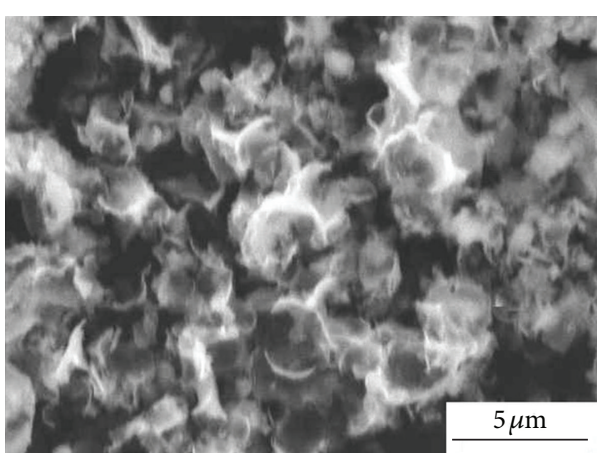

(a)

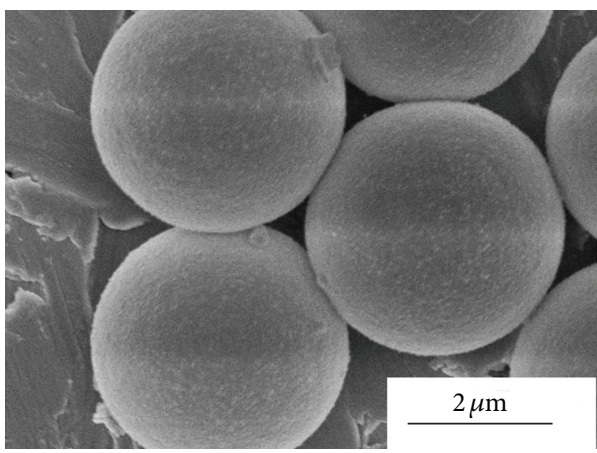

(c)

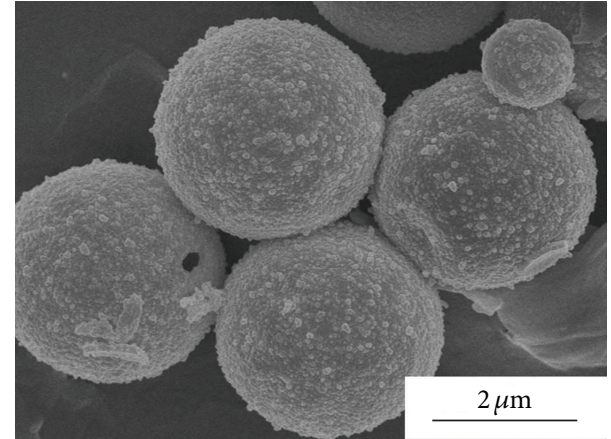

(b)

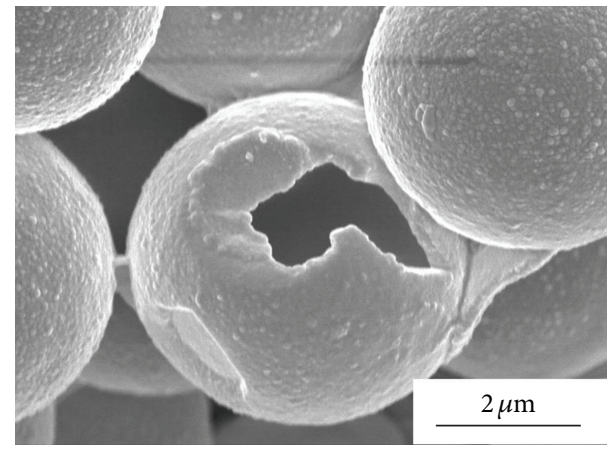

(d)

FigURE 1: SEM images of hollow titania spheres under different TBOT volumes: (a) $0.5 \mathrm{~g}$; (b) $1.0 \mathrm{~g}$; (c) $1.5 \mathrm{~g}$, and (d) $2.0 \mathrm{~g}$.

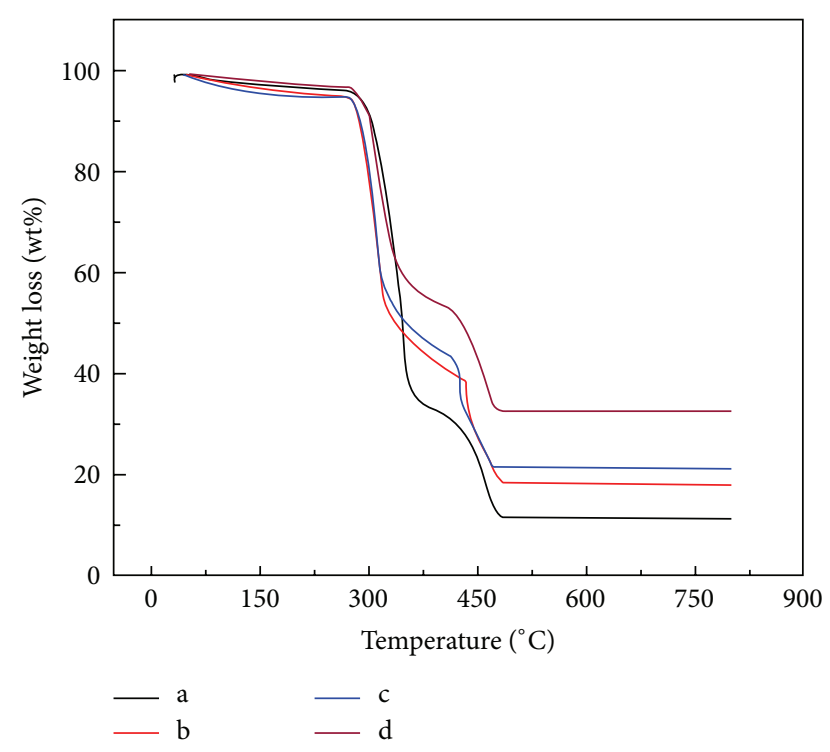

Figure 2: TG curves of the as-synthesized PSA/TiO 2 composite spheres under different TBOT volumes: (a) $0.5 \mathrm{~g}$; (b) $1.0 \mathrm{~g}$; (c) $1.5 \mathrm{~g}$, and (d) $2.0 \mathrm{~g}$. The composite spheres were heated from the ambient temperature to $800^{\circ} \mathrm{C}$ at a rate of $5^{\circ} \mathrm{C} /$ min under oxygen purge.

$400 \sim 4000 \mathrm{~cm}^{-1}$. The sample was prepared by the usual $\mathrm{KBr}$ pellet method. The purity and composition of the products were characterized by powder XRD with D8 Advance X-ray diffractometer ( $\mathrm{Cu} \mathrm{Ka}, \lambda=0.02 \mathrm{~nm}, 30 \mathrm{~mA}, 40 \mathrm{kV})$.

\section{Results and Discussion}

In the previous study, they have developed a facile method to fabricate a series of monodisperse micrometer PSA template spheres in dispersion polymerization [23]. The PSA spheres were flexibly controlled in diameter by altering the initial water volume and the crosslinking agent EGDMA concentration, which simultaneously possessed the favorable surface topography. In the current study, the employment of monodisperse PSA template sphere was a key to the selfassembly of titania spheres into homogeneous crystalline arrays with large domain size. The water vapor-induced internal hydrolysis method was reported to fabricate HTS based on the templating method. In addition, the procedure using TBOT precursor was adopted to ensure coating uniformity.

Figure 1 showed a series of coating reactions in which the TBOT concentration was varied on a scale of $0.5 \mathrm{~g}$ in glass vials to establish the concentration ranges in which HTS were formed without aggregation. The volume of ethanol, and PSA spheres remained constant in all the coating reactions (1.5 g PSA, $20 \mathrm{~mL}$ ethanol and reaction time $15 \mathrm{~h}$ ). When less than $0.5 \mathrm{~g}$ of TBOT was used, there was no spherical structure as exemplified by Figure 1(a). It demonstrated that TBOT was not enough to coat the PSA template spheres to form titania shell. When the amount of TBOT was increased to $1.0 \mathrm{~g}$, the spherical structure appeared as shown in Figure 1(b). Figure 1(c) showed the SEM image of HTS when the amount was $1.5 \mathrm{~g}$. The HTS were obtained hardly any coagulation and secondary nucleation of titania on the surface of HTS. However, when the TBOT content continued 


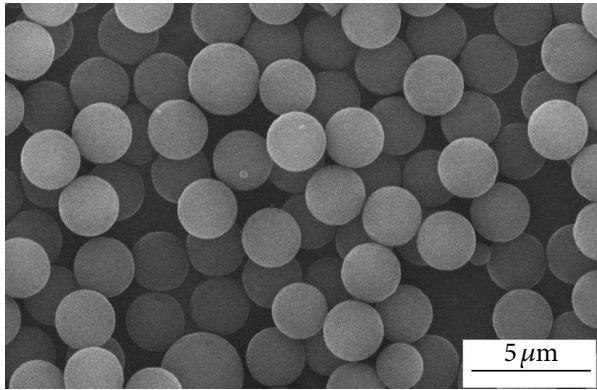

(a)

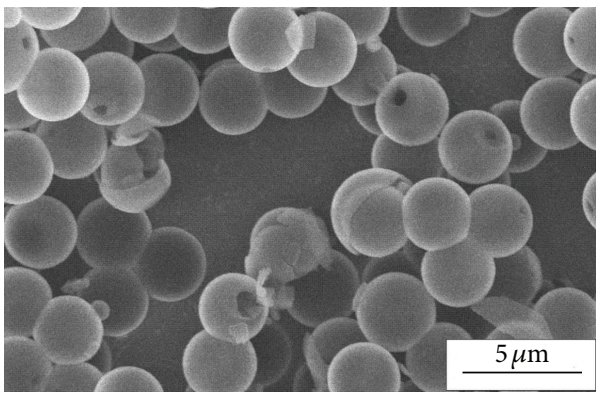

(c)

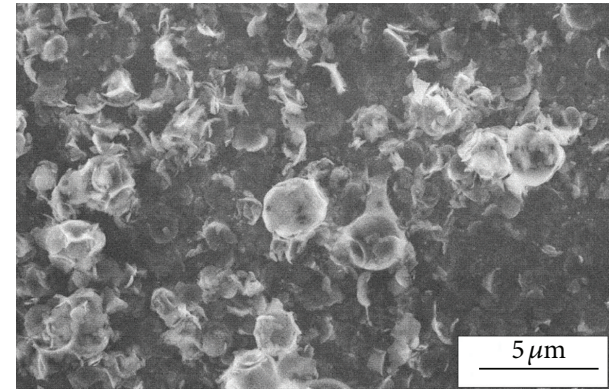

(b)

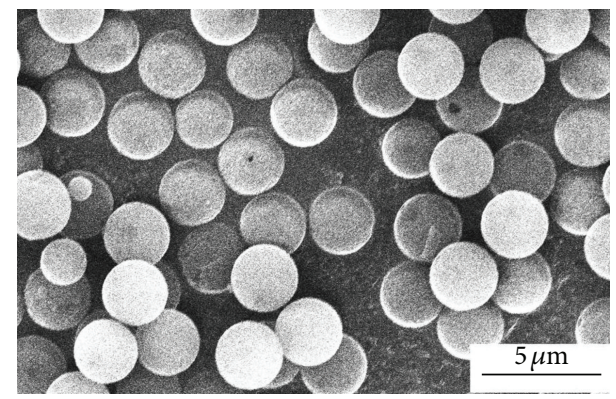

(d)

FIGURE 3: SEM images of hollow titania spheres under the TBOT volume $1.5 \mathrm{~g}$ and different reaction times: (a) PSA template spheres, 0 h; (b) $3 \mathrm{~h}$; (c) $9 \mathrm{~h}$, and (d) $15 \mathrm{~h}$.

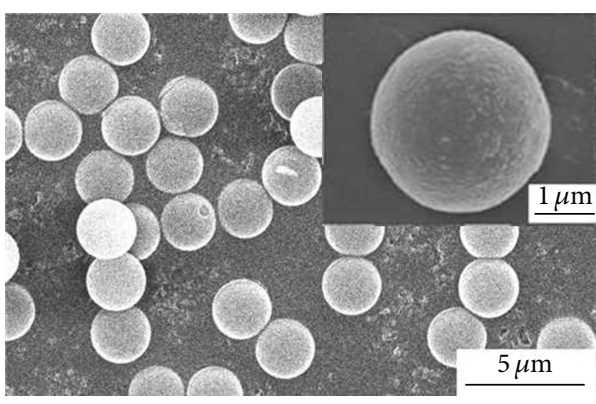

(a)

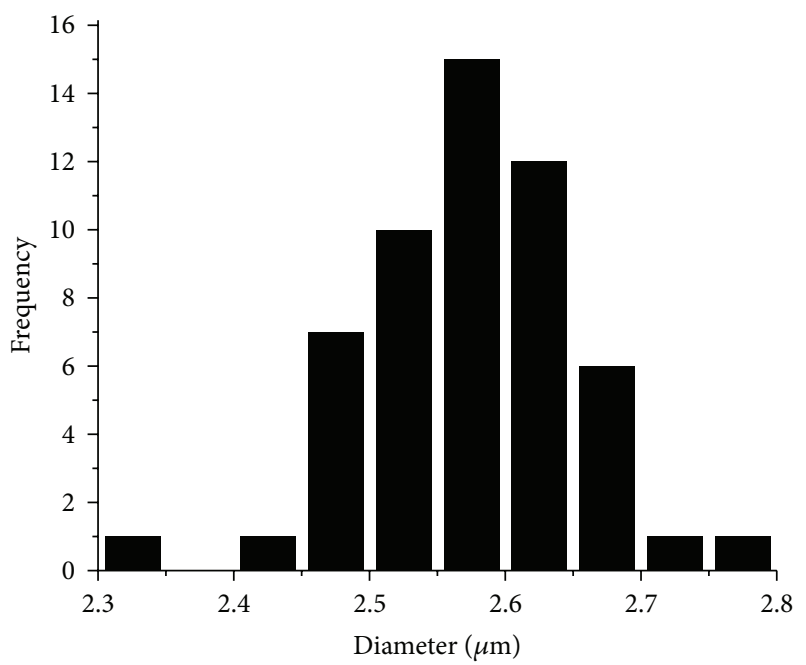

(c)

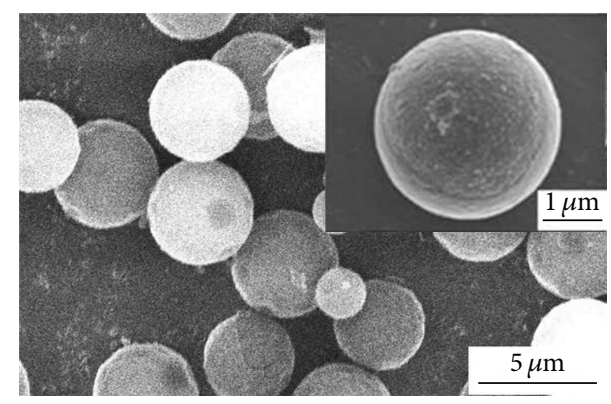

(b)

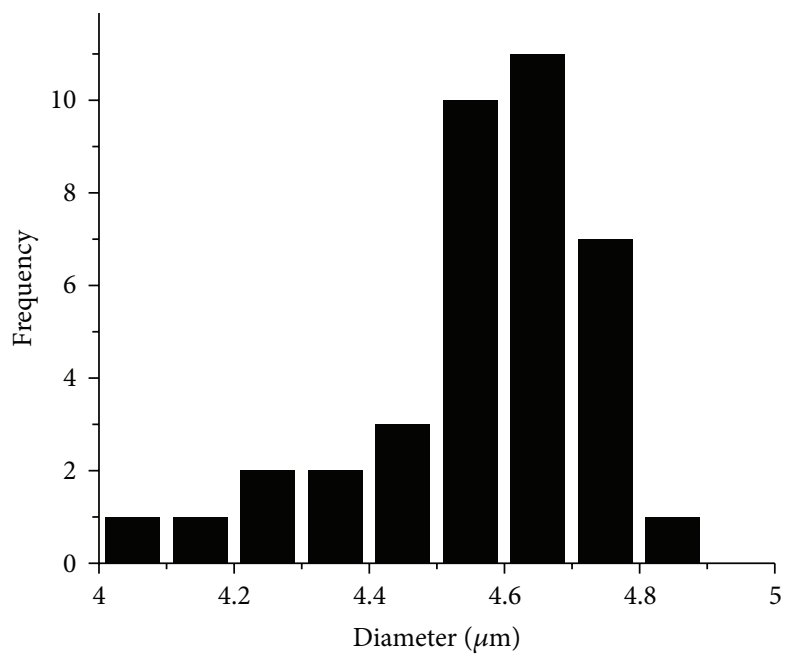

(d)

FIGURE 4: SEM images, FESEM images, and the size distribution histogram of the HTS with different diameters under the reaction time $15 \mathrm{~h}$. 


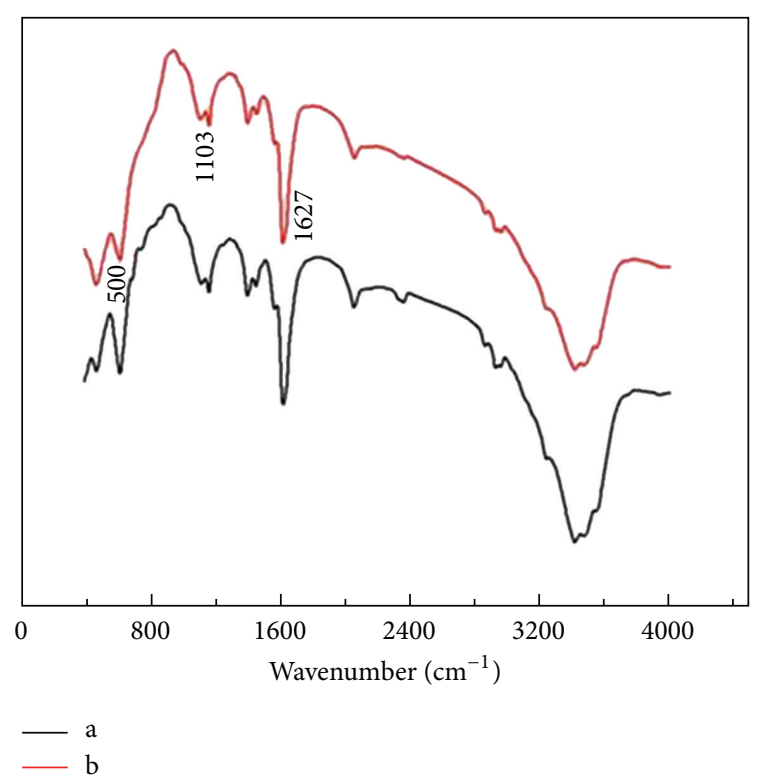

FIGURE 5: FT-IR spectra of (a) PSA/TiO 2 composite spheres dried at $90^{\circ} \mathrm{C}$ and (b) HTS obtained by annealing at $500^{\circ} \mathrm{C}$.

to be increased to $2.0 \mathrm{~g}$, HTS were disfigured and a lot of secondary nucleation of titania could be observed (Figure 1(d)). The reason was that the faster hydrolysis and condensation of TBOT as a result of higher TBOT content resulted in the nucleation of secondary titania particles. Simultaneously, the generated titania nanoparticles did not maintain stable and severely aggregated in solution to deposit onto the surface of PSA template spheres. In this case, the shell thickness of the HTS was also confined by the concentration of TBOT which contrarily decreased the shell thickness of HTS with increasing the TBOT content [25].

The compositions of $\mathrm{PSA} / \mathrm{TiO}_{2}$ composite core-shell spheres were investigated by TG curves as shown in Figure 2. According to the TG curves, the $\mathrm{PSA} / \mathrm{TiO}_{2}$ composite spheres eliminated most of their weight in the temperature range of $270 \sim 320^{\circ} \mathrm{C}$. The evaporation of physically adsorbed water, the desorption of physisorbed template molecules, and the evaporation of residual solvent in the sample occurred in turn. The later weight loss was induced by the oxidative decomposition of PSA template spheres. The PSA cores were completely removed at about $500^{\circ} \mathrm{C}$ and the residual titania accounted for about $11 \%, 18 \%, 21 \%$, and $32 \%$ in proportion corresponding to the Figures 2(a), 2(b), 2(c), and 2(d), respectively. On the other hand, the results revealed that the shell thickness of HTS was increased with the addition of TBOT content in specified concentration range.

The effect of reaction time could be observed using three different reaction times $(3 \mathrm{~h}, 9 \mathrm{~h}$, and $15 \mathrm{~h})$, while other process parameters remained the same $(1.5 \mathrm{~g}$ TBOT, $1.5 \mathrm{~g}$ PSA, and $20 \mathrm{~mL}$ ethanol). Figure 3 showed the SEM of PSA template spheres and HTS at three different times. Figure 3(a) displayed the PSA template spheres with controllable micrometer-sized diameters and consistent spherical feature. In the shorter reaction time $(3 \mathrm{~h})$, the prepared titania

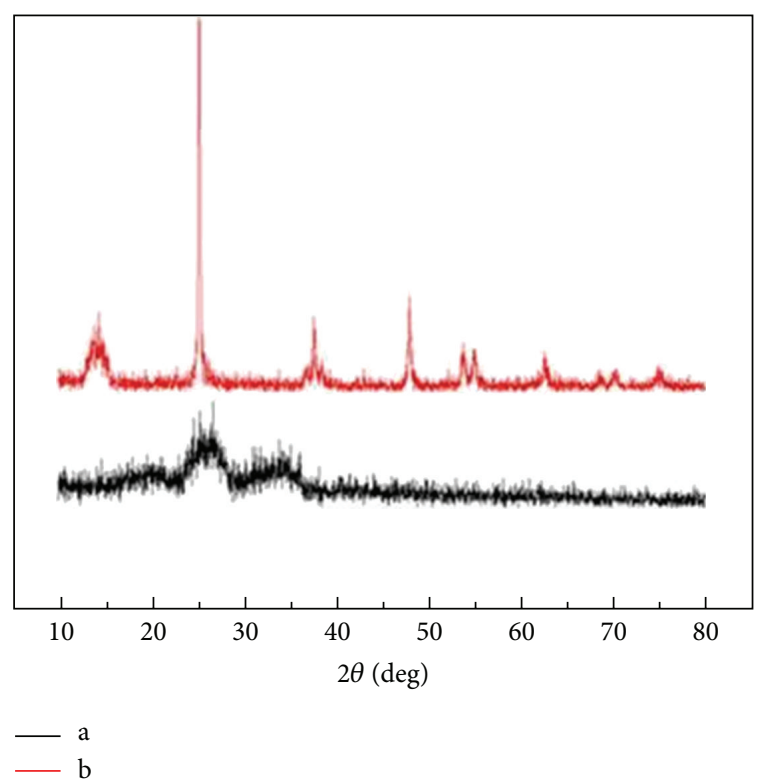

FIGURE 6: XRD patterns of (a) $\mathrm{PSA} / \mathrm{TiO}_{2}$ composite spheres dried at $90^{\circ} \mathrm{C}$ and (b) HTS obtained by annealing at $500^{\circ} \mathrm{C}$.

was irregular particles, and there were few of spherical structure with some residual precursor chemicals as exemplified by Figure 3(b), which indicated that there was only a bit of TBOT to be hydrolyzed, leading to the incomplete coating in the sol-gel process. As the reaction time was increased to $9 \mathrm{~h}$, a lot of spherical titania particles were observed in Figure 3(c). When the reaction time was further increased to $15 \mathrm{~h}$, the titania particles became more spherical as observed in Figure 3(d). Furthermore, the size of HTS was uniform as indicated by the size distribution histogram (Figures 4(c) and $4(\mathrm{~d})$ ). Based on these results, although the precipitation of titania occurred in a short time, it was $15 \mathrm{~h}$ at least to ensure that the titania nanoparticles were completely coated on PSA template spheres. It was beneficial for the formation of uniform HTS with favorable surface topography as shown in the inset FESEM images (Figure 4).

As exemplified by the intentionally broken sample (data not shown), it indeed showed a vivid hollow structure with hollow cavity. The approximate shell thickness was also observed at ca. $150 \mathrm{~nm}$, which could be controlled by changing the amount of TBOT in sol-gel process. The mean shell thickness estimated increased from ca. $100 \mathrm{~nm}$ to $200 \mathrm{~nm}$, accompanying the molar ratio of TBOT/ethanol which increased from 0.007 to 0.013 , respectively. Figure 4 showed different sizes of HTS with controllable diameters and favorable surface topography. The HTS were uniform observed by the size distribution histogram (Figures 4(c) and $4(d)$ ) and the surface topography was commendable as shown in the inset FESEM images.

FT-IR spectrum (Figure 5) of titania spheres had broad bands between 3200 and $3600 \mathrm{~cm}^{-1}$, indicating the presence of hydroxyl groups on the titania surface. Two peaks appeared at approximately 3403 and $1632 \mathrm{~cm}^{-1}$, which were characteristic of stretching and bending vibrations of water molecules, 


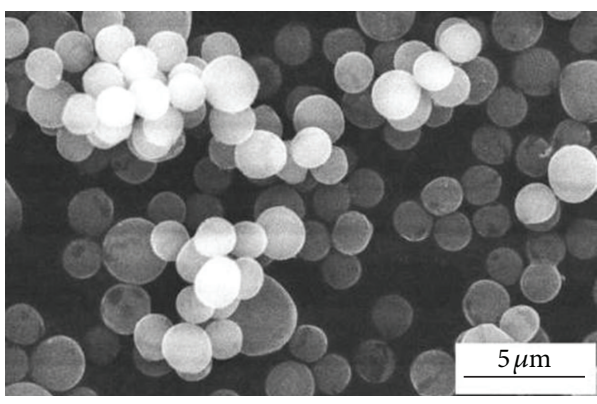

(a)

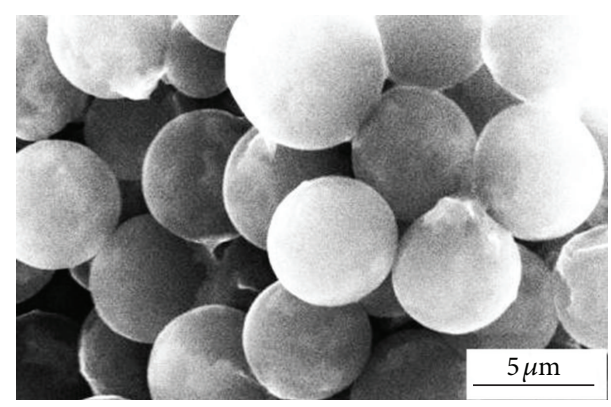

(b)

FIGURE 7: SEM images of the hollow zirconia spheres with different diameters: (a) $1.6 \mu \mathrm{m}$ and (b) $5.5 \mu \mathrm{m}$.

revealing the presence of water molecules adsorbed on the dried titania spheres. A weaker absorption peak at $1401 \mathrm{~cm}^{-1}$ revealed the stretching vibration of little residual organic groups. Both the peak at $1103 \mathrm{~cm}^{-1}$ and the strong peaks near $1627 \mathrm{~cm}^{-1}$ agreed with the Ti-O bond which implied the asymmetric stretching vibration and the anatase phase of titania, respectively. The band at $500 \mathrm{~cm}^{-1}$ was associated with the Ti-O-Ti stretching mode of titania [26].

Figure 6 showed the XRD results of $\mathrm{PSA} / \mathrm{TiO}_{2}$ composite spheres and corresponding HTS after thermal treatment. The results showed that as-prepared $\mathrm{PSA} / \mathrm{TiO}_{2}$ composite spheres had no diffraction peaks, indicating that the powder obtained was amorphous (Figure 6(a)). After heat treatment at $500^{\circ} \mathrm{C}$, the HTS were turned into anatase phase from amorphous phase (Figure $6(\mathrm{~b})$ ). The calcination at $500^{\circ} \mathrm{C}$ not only removed the template but also made the formation of anatase phase [26]. There were no other peaks observed, indicating that the structured HTS were of high purity.

In addition, to further verify the viability of this approach, we also successfully fabricated hollow zirconia spheres using preformed negatively charged PSA particles as template spheres and $\mathrm{ZrOCl}_{2} \cdot 8 \mathrm{H}_{2} \mathrm{O}$ as zirconia precursor. Figure 7 showed different diameters of controllable monodisperse micrometer hollow zirconia spheres. The previous results demonstrated that the approach could be easily extended to the preparation of other metal oxide hollow spheres.

\section{Conclusion}

In summary, a simple and easily scalable approach has been offered for producing well-defined, size tunable HTS by means of modified sol-gel condensation method in ethanol solvent. In this process, the water vapor promoted the hydrolysis of TBOT to form titania coating spheres, which was very simple and achievable. Besides, this approach offered a great deal of control on the diameter and the shell thickness of HTS. We believed that this method could be extended to prepare other inorganic hollow spheres and broaden a variety of applications in magnetics, electronics, photonic crystals, and heat preservation material.

\section{References}

[1] A. Hanprasopwattana, S. Srinivasan, A. G. Sault, and A. K. Datye, "Titania coatings on monodisperse silica spheres (characterization using 2-propanol dehydration and TEM)," Langmuir, vol. 12, no. 13, pp. 3173-3179, 1996.

[2] F. Caruso, A. S. Susha, M. Giersig, and H. Möhwald, "Magnetic core-shell particles: preparation of magnetite multilayers on polymer latex microspheres," Advanced Materials, vol. 11, no. 11, pp. 950-953, 1999.

[3] M. Ocana, W. P. Hsu, and E. Matijevic, "Preparation and properties of uniform-coated colloidal particles. 6: titania on zinc oxide," Langmuir, vol. 7, no. 12, pp. 2911-2916, 1991.

[4] A. Imhof and D. J. Pine, "Ordered macroporous materials by emulsion templating," Nature, vol. 389, no. 6654, pp. 948-951, 1997.

[5] B. T. Holland, C. F. Blanford, and A. Stein, "Synthesis of macroporous minerals with highly ordered three-dimensional arrays of spheroidal voids," Science, vol. 281, no. 5376, pp. 538-540, 1998.

[6] F. Caruso, R. A. Caruso, and H. Möhwald, "Nanoengineering of inorganic and hybrid hollow spheres by colloidal templating," Science, vol. 282, no. 5391, pp. 1111-1114, 1998.

[7] K. Y. Chen and Y. W. Chen, "Synthesis of spherical titanium dioxide particles by homogeneous precipitation in acetone solution," Journal of Sol-Gel Science and Technology, vol. 27, no. 2, pp. 111-117, 2003.

[8] A. Imhof, "Preparation and characterization of titania-coated polystyrene spheres and hollow titania shells," Langmuir, vol. 17, no. 12, pp. 3579-3585, 2002.

[9] Y. Lu, H. Fan, A. Stump, T. L. Ward, T. Rieker, and C. J. Brinker, "Aerosol-assisted self-assembly of mesostructured spherical nanoparticles," Nature, vol. 398, no. 6724, pp. 223-226, 1999.

[10] S. Schacht, Q. Huo, I. G. Voigt-Martin, G. D. Stucky, and F. Schüth, "Oil-water interface templating of mesoporous macroscale structures," Science, vol. 273, no. 5276, pp. 768-771, 1996.

[11] J. Hotz and W. Meier, "Vesicle-templated polymer hollow spheres," Langmuir, vol. 14, no. 5, pp. 1031-1036, 1998.

[12] B. M. Discher, Y. Y. Won, D. S. Ege et al., "Polymersomes: tough vesicles made from diblock copolymers," Science, vol. 284, no. 5417, pp. 1143-1146, 1999.

[13] T. von Werne and T. E. Patten, "Atom transfer radical polymerization from nanoparticles: a tool for the preparation of 
well-defined hybrid nanostructures and for understanding the chemistry of controlled/"living" radical polymerizations from surfaces," Journal of the American Chemical Society, vol. 123, no. 31, pp. 7497-7505, 2001.

[14] R. A. Caruso, A. Susha, and F. Caruso, "Multilayered titania, silica, and Laponite nanoparticle coatings on polystyrene colloidal templates and resulting inorganic hollow spheres," Chemistry of Materials, vol. 13, no. 2, pp. 400-409, 2001.

[15] F. Caruso, X. Y. Shi, R. A. Caruso, and A. Susha, "Hollow titania spheres from layered precursor deposition on sacrificial colloidal core particles," Advanced Materials, vol. 13, no. 10, pp. 740-744, 2001.

[16] M. L. Breen, A. D. Dinsmore, R. H. Pink, S. B. Qadri, and B. R. Ratna, "Sonochemically produced ZnS-coated polystyrene core-shell particles for use in photonic crystals," Langmuir, vol. 17, no. 3, pp. 903-907, 2001.

[17] K. P. Velikov and A. van Blaaderen, "Synthesis and characterization of monodisperse core-shell colloidal spheres of zinc sulfide and silica," Langmuir, vol. 17, no. 16, pp. 4779-4786, 2001.

[18] H. Ihara, S. Kubota, A. Uchimura et al., "A facile preparation method for self-assembled monolayers with silica particles on polystyrene-based microspheres," Materials Chemistry and Physics, vol. 114, no. 1, pp. 1-5, 2009.

[19] A. Rogach, A. Susha, F. Caruso et al., "Nano- and microengineering: 3-D colloidal photonic crystals prepared from sub- $\mu \mathrm{m}$ sized polystyrene latex spheres pre-coated with luminescent polyelectrolyte/nanocrystal shells," Advanced Materials, vol. 12, no. 5, pp. 333-337, 2000.

[20] O. Kalinina and E. Kumacheva, "Polymeric nanocomposite material with a periodic structure," Chemistry of Materials, vol. 13, no. 1, pp. 35-38, 2001.

[21] X. L. Xu and S. A. Asher, "Synthesis and utilization of monodisperse hollow polymeric particles in photonic crystals," Journal of the American Chemical Society, vol. 126, no. 25, pp. 79407945, 2004.

[22] J. X. Wang, L. X. Wen, Z. H. Wang, and J. F. Chen, "Immobilization of silver on hollow silica nanospheres and nanotubes and their antibacterial effects," Materials Chemistry and Physics, vol. 96, no. 1, pp. 90-97, 2006.

[23] M. Z. Xu, W. Z. Li, M. X. Du et al., "A facile method to fabricate a series of micrometer scale hollow silica spheres," Materials Letters, vol. 64, no. 8, pp. 931-934, 2010.

[24] M. Z. Xu, M. X. Du, L. M. Zhao et al., "Facile preparation of monodisperse micrometer-sized hollow silica spheres with tunable size and commendable surface topography," Materials Research Bulletin, vol. 45, no. 9, pp. 1056-1063, 2010.

[25] X. Cheng, M. Chen, L. Wu, and G. Gu, "Novel and facile method for the preparation of monodispersed titania hollow spheres," Langmuir, vol. 22, no. 8, pp. 3858-3863, 2006.

[26] J. Yin, L. He, Z. Gao, L. Gao, and B. Wang, "Facile method for fabricating titania spheres for chromatographic packing," Materials Letters, vol. 63, no. 26, pp. 2191-2193, 2009. 

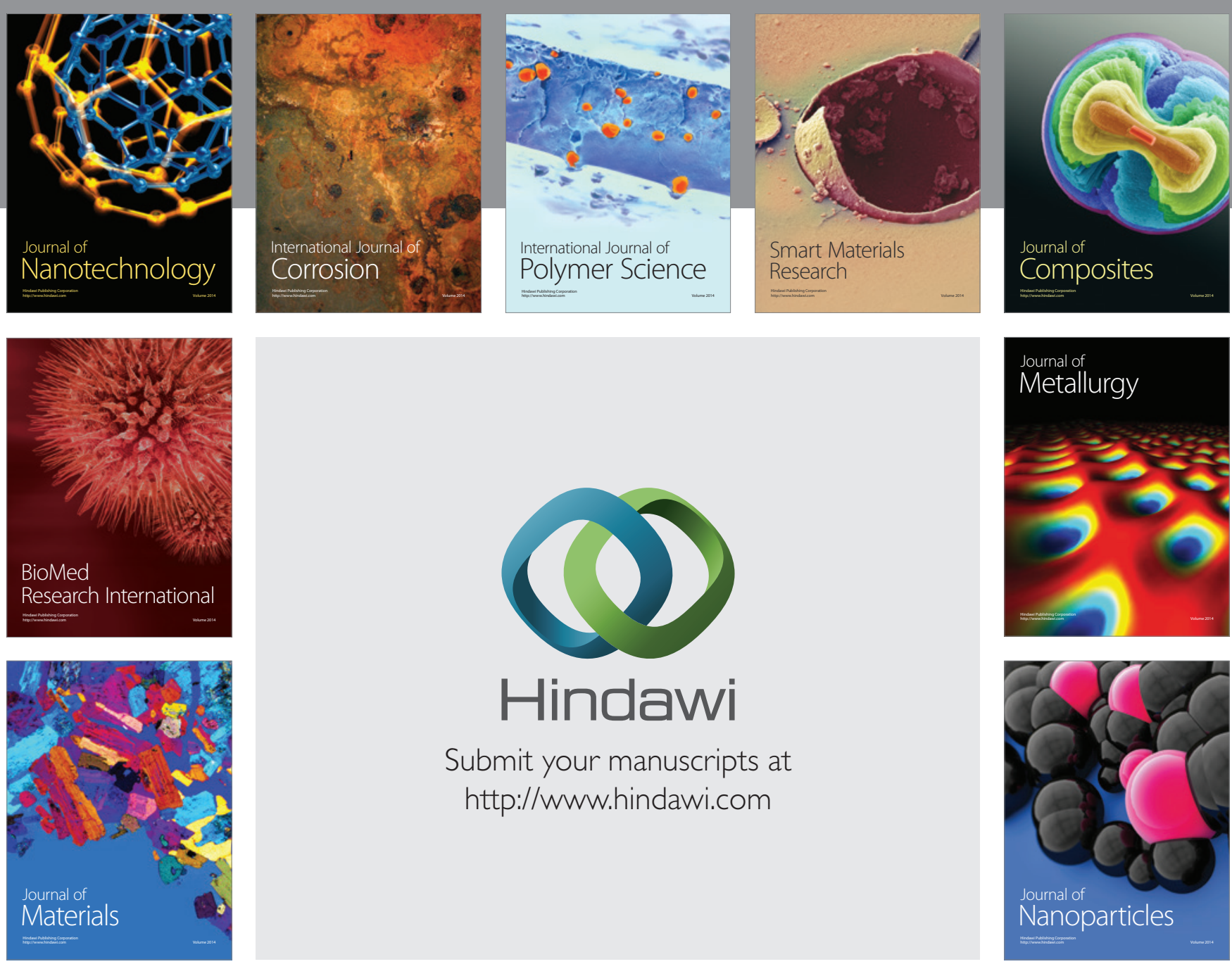

Submit your manuscripts at http://www.hindawi.com
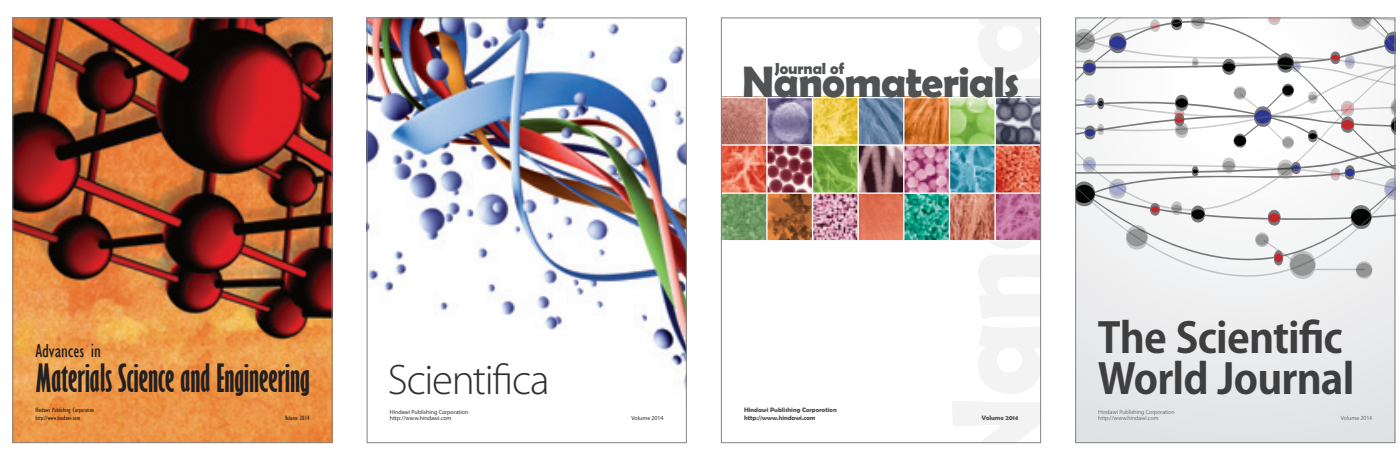

\section{The Scientific World Journal}
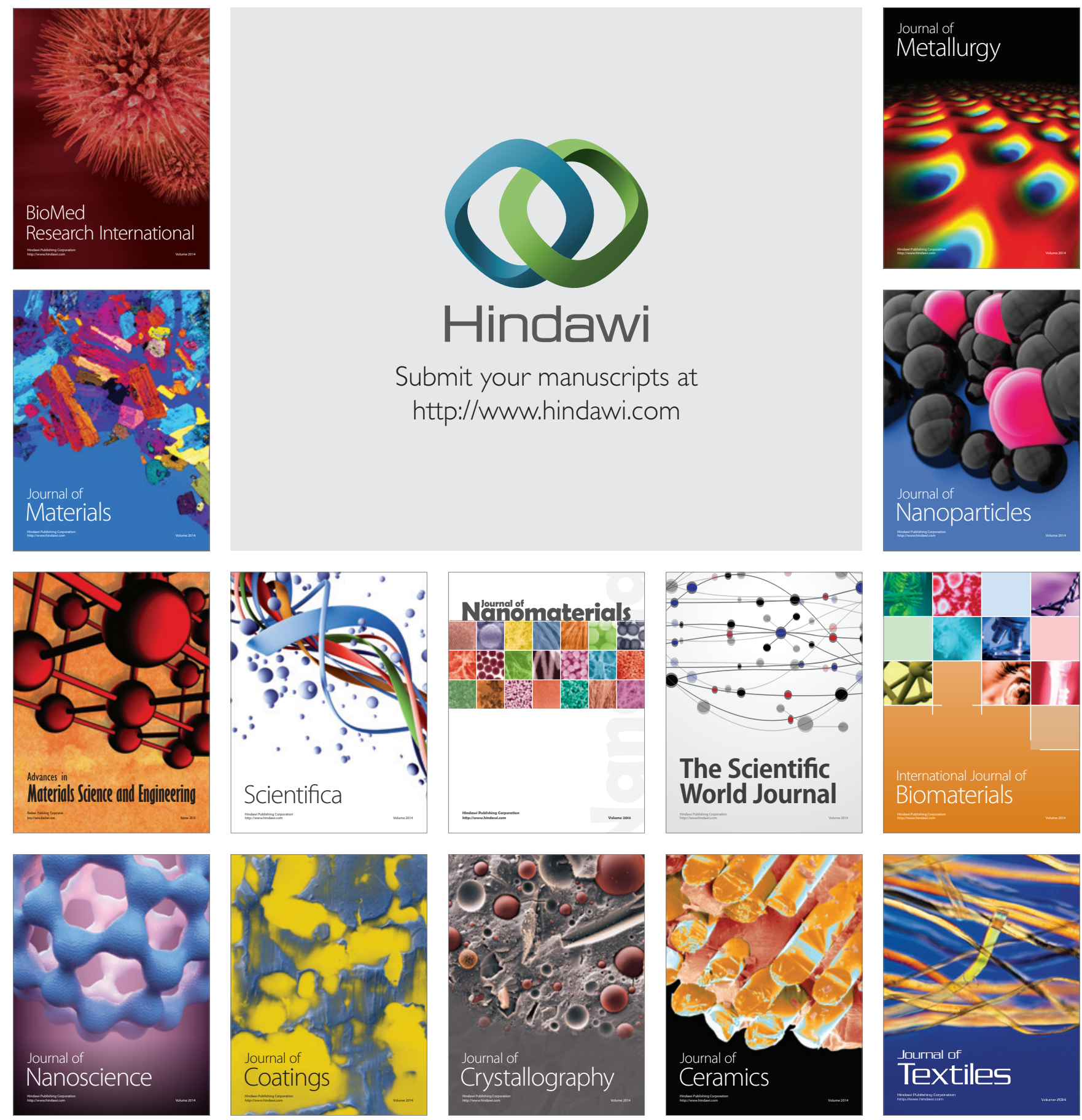